Nervenarzt 2014 • 85:667-668

DOI 10.1007/s00115-014-4071-1

Online publiziert: 17. Mai 2014

๑) Springer-Verlag Berlin Heidelberg 2014

W. Hacke' $\cdot$ W. Maier ${ }^{2} \cdot$ C. Braun ${ }^{3}$

${ }^{1}$ Neurologische Klinik, Universität Heidelberg

${ }^{2}$ Klinik für Psychiatrie und Psychotherapie der Universität Bonn

${ }^{3}$ Springer-Verlag, Heidelberg

\title{
Professor Brandt als Herausgeber verabschiedet
}

\section{Prägte 25 Jahre die Zeitschrift Der Nervenarzt}

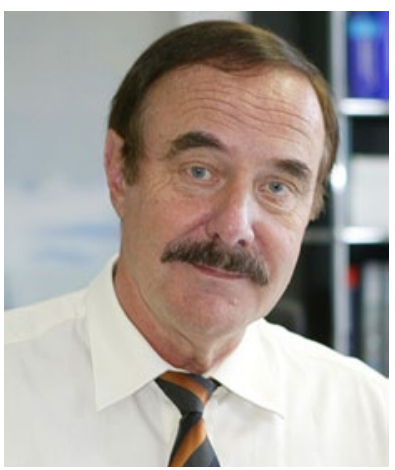

Im Februar hat sich Professor Brandt als federführender Herausgeber von Der Nervenarzt aus dem Board der Zeitschrift zurückgezogen. 25 Jahre lang hat er die Zeitschrift maßgeblich geprägt, zunächst als Herausgeber, seit 1998 als federführender Herausgeber Neurologie.

Bei seiner Berufung in das Herausgeber-Board war Brandt Leiter der Klinik für Neurologie am Klinikum Großhadern der Ludwig-Maximilians-Universität München. Schon als Assistenzarzt in Freiburg - nach dem Medizinstudium in Köln und einem kurzen Ausflug in die Neurochirurgie - widmete sich Brandt seinen späteren Kernthemen: Optokinetik und vestibulärer Schwindel. 1976 wechselte er als Chefarzt der Neurologischen Klinik des Alfred-Krupp-Krankenhauses nach Essen.

Während seiner Tätigkeit in München baute Brandt ein national wie international angesehenes integriertes Forschungsund Behandlungszentrum für Schwindel, Gleichgewichts- und Augenbewegungsstörungen auf. Seit 2006 widmet er sich als Inhaber der ersten Senior-Forschungsprofessur der Hertie-Stiftung ausschließlich der Forschung.
Trotz beruflicher Verpflichtungen als Leiter einer großen Klinik, intensiver, international beachteter Forschungstätigkeit und seinem Engagement in verschiedenen Fachgesellschaften, unter anderem der Deutschen Gesellschaft für Neurologie und der European Neurological Society, deren Vorsitz er 1997 bis 1998 bzw. 1998 bis 1999 inne hatte, war Professor Brandt von Anfang an ein unverzichtbarer Mitgestalter von Der Nervenarzt.

Die 25 Jahre, in denen er die Geschicke der Zeitschrift mitbestimmte, waren geprägt von Veränderungen und $\mathrm{He}$ rausforderungen. An erster Stelle ist hier die Digitalisierung der Inhalte zu nennen. War es anfangs allein das gedruckte Heft, über das Der Nervenarzt die Leser erreichte, so wurden ab 1996 alle Inhalte konsequent in einem Online-Archiv zur Verfügung gestellt, wobei Übersichten und Originalarbeiten seit einigen Jahren auch „online first“ publiziert werden. Als Folge dieser Entwicklung ist Der Nervenarzt heute weiter verbreitet als je zuvor. Mehr als 8500 Institute und Kliniken können inzwischen weltweit auf die Online-Version zugreifen und tun dies auch. Knapp 200.000 Volltext-Downloads pro Jahr belegen dies eindrucksvoll.

Seit 2002 erscheinen in der Rubrik „CME Zertifizierte Fortbildung "State-of-the-ArtArtikel, die in einem 4-Jahres-Turnus alle wichtigen Gebiete der Neurologie und Psychiatrie mit deutlichem Praxisbezug abdecken. Das Fortbildungskonzept wurde mit der Einführung von Leitthemen einige Jahre später konsequent weiterentwickelt.

Einen weiteren Schritt in der Nutzung neuer Techniken ging Der Nervenarzt 2011, 
als mit dem Editorial Manager ${ }^{\circledR}$ ein elektronisches Manuskriptverwaltungssystem eingeführt wurde. Seither können Autoren ihre Manuskripte bequem online einreichen. Dies erlaubte gleichzeitig, den gestiegenen Anforderungen an die Begutachtung wissenschaftlicher medizinischer Beiträge gerecht zu werden. Das Peer-ReviewVerfahren wurde als integraler Bestandteil bei Der Nervenarzt professionalisiert.

Die Ehrungen aufzuzählen, die Prof. Brandt für seine außergewöhnliche Lebensleistung zuteil wurden, würde den Rahmen sprengen. Vielmehr möchten sich die federführenden Herausgeber von Der Nervenarzt sowie Geschäftsführung und Redaktion des Springer-Verlags auf das herzlichste bei Prof. Brandt bedanken. Er hat wesentlich dazu beigetragen, dass Der Nervenarzt auf eine erfolgreiche Geschichte zurückblickt und gut gerüstet in eine spannende Zukunft geht.

„Ein guter Tag", so Brandt in einem Interview mit der Frankfurter Allgemeinen Zeitung, „beginnt mit der Freude auf die bevorstehenden Projekte". In diesem Sinne wünschen wir Prof. Brandt viel Inspiration, Schaffenskraft, Gesundheit und Glück.

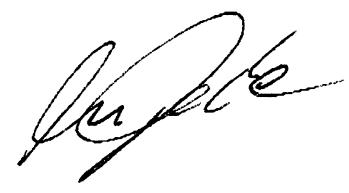

Prof. Dr. Dr. h.c. Werner Hacke

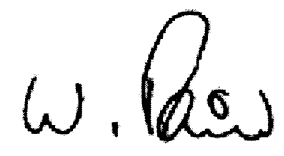

Prof. Dr. Wolfgang Maier

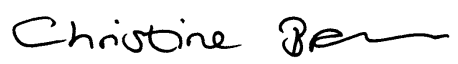

Christine Braun

\section{Korrespondenzadresse}

Prof. Dr. Dr. h.c. W. Hacke

Neurologische Klinik, Universität Heidelberg Im Neuenheimer Feld 400, 69120 Heidelberg werner.hacke@med.uni-heidelberg.de

Interessenkonflikt. W. Hacke, W. Maier und C. Braun geben an, dass kein Interessenkonflikt besteht.

\section{springermedizin.de - komfortabel recherchieren in der e.Bibliothek}

Ohne Umwege zur gewünschten Information zu gelangen - springermedizin.de macht's möglich. Verzichten Sie auf überquellende Stehsammler und unübersichtliche Papierstapel, und nutzen Sie statt dessen die digitale e.Bibliothek von Springer Medizin.

Suchen Sie Beiträge in einer bestimmten Fachzeitschrift Ihres Fachgebiets? Oder möchten Sie englischsprachige Journals für eine interdisziplinäre Recherche nutzen? Interessieren Sie sich für Übersichtsbeiträge oder aktuelle wissenschaftliche Studien?

Die e.Bibliothek wird all diesen Anforderungen gerecht: Sie umfasst über 600 deutschsprachige und internationale Fachzeitschriften aus allen Bereichen der Medizin inklusive der medizinischen Inhalte von SpringerLink.

Die e.Bibliothek beinhaltet auch „Online First"-Beiträge, also hoch aktuelle Beiträge, die bereits vor Erscheinen einer gedruckten Ausgabe in elektronischer Form publiziert wurden.

\section{Komfortable und schnelle} Recherche

- Mit der Volltextsuche von springermedizin.de durchsuchen Sie den gesamten Inhalt der e.Bibliothek und gelangen direkt zu den Inhalten, die für Sie relevant sind.

- AufWunsch können Sie die Suchergebnisse eingrenzen und beispielsweise gezielt in einzelnen Zeitschriften, nach Themen und sogar nach Autoren suchen.

- Wenn Sie einen englischen Suchbegriff eingeben, erhalten Sie zudem Treffer aus den englischsprachigen wissenschaftlichen Zeitschriften von SpringerLink.
Mit der Suchfunktion gelangen Sie auch zu Inhalten aus allen anderen Bereichen von springermedizin.de von zertifizierten Fortbildungskursen der e.Akademie, aktuellen Kongressberichten bis hin zu aktuellen Themendossiers, Videos und Nachrichten aus der Gesundheits- und Berufspolitk.

\section{Ihre persönliche Merkliste}

Finden Sie einen Beitrag besonders interessant oder möchten Sie ihn für die spätere Lektüre vormerken? Auf springermedizin.de können Sie ganz einfach Ihre persönliche Merkliste anlegen: ein Klick auf das Symbol "merken“ am Beitragsende genügt und die Beiträge erscheinen unter "Meine Merkliste".

Alle Beiträge sind als PDF-Datei im Layout der gedruckten Ausgabe sowie als HTML-Version verfügbar. In der HTMLVersion können Sie die Vorteile der verlinkten Literatur nutzen und direkt zu den zitierten Quellen gelangen.

e.Med - der Zugang zu allen digitalen Inhalten von Springer Medizin

Zugang zu allen Inhalten der e.Bibliothek bekommen Sie mit e.Med. Lernen Sie die Vorzüge dieses umfassenden Angebots kennen und testen Sie e.Med 30 Tage lang kostenlos und unverbindlich unter

\section{www.springermedizin.de/eMed}

Eine erfolgreiche Recherche wünscht Ihnen Ihr Redaktionsteam Fachzeitschriften von Springer Medizin 